\title{
Índice de desenvolvimento sustentável municipal: aplicação na região Costa do Cacau - BA
}

\author{
Elizabeth Santos de Oliveira ${ }^{1 \star(D)}$, Fabio Antonio da Silva Reis ${ }^{1}$ (D), Eurisa Maria de Santana ${ }^{1}$ (D), Christiana \\ Cabiciere Profice $^{1}$ (D), Franco Dino Rico Amado ${ }^{1}$ (D), Ana Maria Souza dos Santos Moreau ${ }^{1}$ (D) \\ ${ }^{1}$ Programa de Pós-Graduação em Desenvolvimento e Meio Ambiente, Universidade Estadual de Santa Cruz, Campus \\ Soane Nazaré de Andrade, Pavilhão Jorge Amado, Rodovia Jorge Amado, Km 16, Bairro Salobrinho, Ilhéus, Bahia, \\ 45662-900. \\ *Autor para correspondência: olivrelizabeth@outlook.com
}

Recebido em 18 de abril de 2020.

Aceito em 27 de setembro de 2020.

Publicado em 30 de setembro de 2020.

Resumo - A utilização de indicadores facilita tomadas de decisões e medição de desempenho de políticas de sustentabilidade. Nesse contexto, esse estudo teve como objetivo levantar indicadores das dimensões social, econômica, ambiental e institucional, para gerar o índice de sustentabilidade municipal (IDSM) e verificar o desenvolvimento socioeconômico e ambiental dos municípios da região turística Costa do Cacau do estado da Bahia. Dentre os municípios analisados, apenas Ilhéus apresentou o nível de sustentabilidade municipal considerado ideal, Itabuna e Ipiaú apresentaram IDSM aceitável, e os demais apresentaram IDMS ao nível de alerta. Este fato evidencia a necessidade urgente de implementação de políticas públicas em todas as dimensões abordadas, de modo a elevar o nível de sustentabilidade e proporcionar condições adequadas de qualidade de vida à população atual e às gerações futuras.

Palavras-chave: Indicadores. Políticas Públicas. Sustentabilidade.

\section{Municipal sustainable development index: application in the Costa do Cacau region - BA}

\begin{abstract}
The use of indicators facilitates decision-making and performance measurement of sustainability policies. In this context, this study aimed to raise indicators of social, economic, environmental, and institutional dimensions, to generate the municipal sustainability index (MSI) and verify the socioeconomic and environmental development of the municipalities in the Costa do Cacau tourist region of the state of Bahia. Among the municipalities analyzed, only Ilhéus presented a level of municipal sustainability considered ideal, while Itabuna and Ipiaú presented acceptable MSI, and the others presented MSI at the alert level. This fact highlights the urgent need to implement public policies in all dimensions addressed, to raise the level of sustainability and provide adequate quality of life conditions for the current population and future generations.
\end{abstract}

Key words: Indicators. Public Policy. Sustainability. 


\section{Índice de desarrollo sostenible municipal: aplicación en la región de Costa do Cacau - BA}

Resumen - El uso de indicadores facilita la toma de decisiones y la medición del desempeño de las políticas de sustentabilidad. En este contexto, este estudio tuvo como objetivo plantear indicadores de las dimensiones social, económica, ambiental e institucional, para generar el índice de sustentabilidad municipal (IDSM) y verificar el desarrollo socioeconómico y ambiental de los municipios de la región turística Costa do Cacau del estado de Bahia. Entre los municipios analizados, solo Ilhéus presentó el nivel de sustentabilidad municipal considerado ideal, Itabuna e Ipiaú presentaron IDSM aceptable, y los demás presentaron IDMS al nivel de alerta. Este hecho resalta la urgente necesidad de implementar políticas públicas en todas las dimensiones abordadas, con el fin de elevar el nivel de sustentabilidad y brindar condiciones adecuadas de calidad de vida a la población actual y las generaciones futuras.

Palabras clave: Indicadores. Política Pública. Sustentabilidad.

\section{Introdução}

O conceito de desenvolvimento sustentável originou-se em meio ao cenário mundial de grandes transformações políticas, econômicas e debates sobre os impactos do crescimento econômico aos ecossistemas naturais durante as décadas de 70 e 80 . Foi publicado pela primeira vez no documento intitulado "Nosso Futuro Comum" em 1987, que o definiu como "desenvolvimento que satisfaz as necessidades presentes, sem comprometer a capacidade das gerações futuras de suprir suas próprias necessidades" (Comissão Mundial Sobre Meio Ambiente e Desenvolvimento 1988, p. 46).

Ao longo dos debates sobre os impactos do crescimento econômico ao ambiente, houve a fortificação do paradigma do desenvolvimento sustentável ocasionando transformações no modelo de desenvolvimento dos países a partir de acordos mundiais. No Brasil, pode-se notar essa influência na adoção e desenvolvimento de leis ambientais. O artigo 225 da Constituição Federal (1988) dispõe que "todos têm direito ao meio ambiente ecologicamente equilibrado, bem de uso comum do povo e essencial à sadia qualidade de vida, impondo-se ao poder público e à coletividade o dever de defendêlo e preservá-lo para as presentes e futuras gerações".

Nota-se que a discussão sobre o desenvolvimento sustentável envolve a problemática econômica, ambiental e social. Para Leff (2004), a crise ambiental é uma crise de conhecimento, sendo imprescindível que a política ambiental seja aplicada a partir da intervenção do Estado, envolvendo ações que contemplem as áreas socioculturais, educacionais e a participação da sociedade civil em tomadas de decisões, tendo em vista um desenvolvimento sustentável e democrático.

Em 1992 foi realizada, no Rio de Janeiro, a Conferência Internacional da Organização das Nações Unidas sobre Meio Ambiente e Desenvolvimento, com a criação da Comissão de Desenvolvimento Sustentável (CDS) e a Agenda 21, como parte dos esforços para transformar o desenvolvimento sustentável em uma meta global aceitável (Bellen 2004). Na Agenda 21 é expressa, nos capítulos 8 e 40, a necessidade de consolidação de indicadores de desenvolvimento sustentável, para isso, a CDS iniciou um projeto de 5 anos (1995-2000) que teve como principal objetivo a construção de indicadores de desenvolvimento sustentável, definindo-os, elucidando metodologias e fornecendo atividades de treinamento e capacitação para sua construção (Malheiros et al. 2008). 
Segundo Resende e Fagundes (2017), no cenário municipal o uso de indicadores pelos gestores públicos é relevante na sinalização das intervenções necessárias para correção das discrepâncias identificadas pelos indicadores. Assim é possível a adoção de medidas que fomentem a sustentabilidade local e, consequentemente regional, atingindo um patamar de sustentabilidade efetiva. Por conta da proximidade da esfera local com os cidadãos e das suas necessidades cotidianas, os municípios estão inseridos no campo privilegiado na promoção da sustentabilidade.

Para Frainer et al. (2017), indicadores são cruciais para guiar os gestores em uma variedade de caminhos na condução das políticas públicas, pois as informações geradas pelos mesmos facilitam a tomada de decisão, auxiliando na mensuração do desempenho das políticas para a sustentabilidade.

No Brasil, oíndice de desenvolvimento sustentável foi desenvolvido pelo IBGE (Instituto Brasileiro de Geografia e Estatística), baseado na definição dos indicadores do movimento internacional, liderado pela Comissão para o Desenvolvimento Sustentável (CDS), das Nações Unidas (Martins e Cândido 2012). A ordenação dos indicadores, realizada pelo IBGE, segue o proposto em 2001 e revisto em 2007 pela Organização das Nações Unidas (ONU), abrangendo as dimensões ambiental, social, econômica e institucional.

A dimensão ambiental trata da preservação e conservação do ambiente, importantes para manutenção da qualidade de vida das gerações atuais e futuras, contemplando os temas atmosfera, terra, água doce, oceanos, mares e áreas costeiras, biodiversidade e saneamento. A dimensão social está relacionada aos objetivos ligados a satisfação das necessidades humanas, a melhoria da qualidade de vida e justiça social, contemplando os temas população, trabalho e rendimento, saúde, educação, habitação e segurança. A dimensão econômica trata das questões de desempenho econômico e financeiro, uso e esgotamento de recursos naturais, produção e gerenciamento de resíduos e uso de energia. A dimensão institucional está relacionada a orientação política, capacidade e esforços realizados pelo governo e sociedade para implementação do desenvolvimento sustentável (IBGE 2004).

Existe uma variedade de Sistemas de Indicadores direcionados a países, no entanto, para espaços geográficos mais específicos, como municípios, os mesmos são escassos ou inexistentes (Martins e Cândido 2012). Atentos a isso, os autores citados desenvolveram a metodologia para IDSM (Índice de Desenvolvimento Sustentável Municipal) a partir da disponibilidade e acesso dos dados para os municípios brasileiros, levando em consideração as características e diversidades locais. Tal metodologia foi à utilizada na presente pesquisa. Diante do exposto, o presente trabalho objetivou analisar o nível do índice de desenvolvimento sustentável (IDS) dos municípios pertencentes à região turística Costa do Cacau, no que tange aos aspectos do desenvolvimento socioeconômico e ambiental.

Os resultados aqui produzidos poderão subsidiar as decisões dos agentes públicos municipais na adoção das medidas necessárias para alcançar cenários exequíveis e desejáveis, visando o equilíbrio entre bem-estar social, econômico e ambiental. O interesse em explorar os indicadores dos municípios da região turística Costa do Cacau partiu da necessidade de analisar de que forma o desenvolvimento econômico, com base no turismo, influencia no desenvolvimento social e ambiental. 


\section{Materiais e métodos}

\section{Área de estudo}

Localizada na mesorregião do Sul Baiano, a Costa do Cacau (Figura 1) compreende os municípios de: Canavieiras, Ilhéus, Itabuna, Itacaré, Ipiaú, Maraú, Pau Brasil, Santa Luzia, São José da Vitória, Una e Uruçuca (Ministério do Turismo 2020).

Com clima tropical e chuvas bem distribuídas durante o ano, solos de boa fertilidade e bioma da Mata Atlântica, a configuração espacial e econômica da Costa do Cacau se caracterizou pela exploração de seus recursos naturais desde o Brasil Colônia, inicialmente com a intensa extração da espécie vegetal arbórea Caesalpina enchinata (Pau-Brasil) e posteriormente, com a implantação de monoculturas de cana-de-açúcar e cacau (Soares et al. 2015). A cultura cacaueira permaneceu e se fortaleceu na região (Bahia 2016), mas nos últimos anos, se projetou com o desenvolvimento do turismo (Soares et al. 2015), passando a ser denominada região turística Costa do Cacau, polo turístico criado em 1995 pela CONDETUR/SUDETUR (Aguiar e Pires 2019). Tal fato ocorreu a partir do incentivo ao desenvolvimento do ecoturismo na década de 80, período em que ocorreu a queda produtiva do cacau ocasionado pela chegada da praga Crinipellis perniciosa (vassoura de bruxa) (Carvalho et al. 2011).

Figura 1. Mapa de Localização da região turística Costa do Cacau - Bahia.
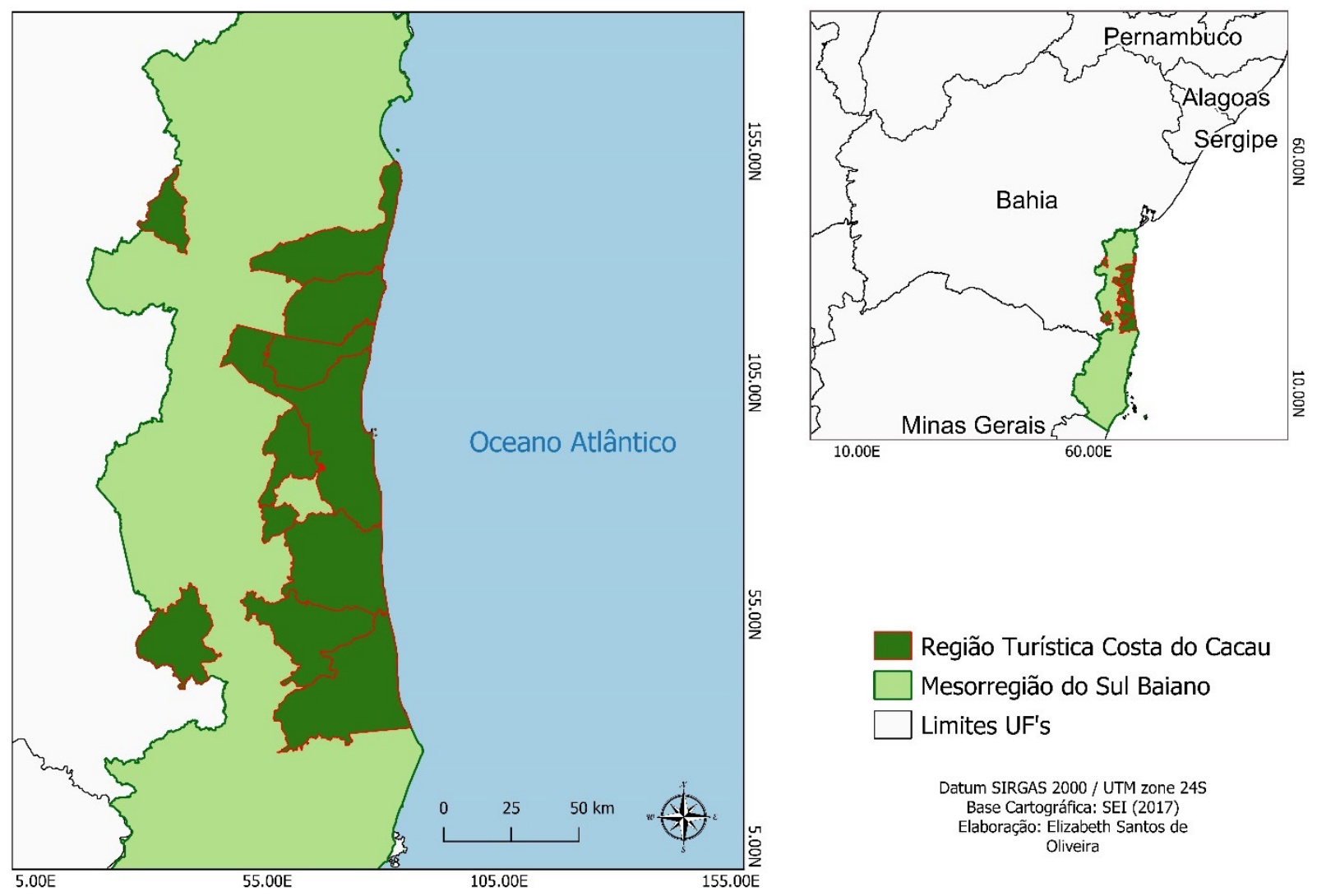

Datum SIRGAS 2000 / UTM zone 24S Base Cartográfica: SEI (2017) Elaboração: Elizabeth Santos de Oliveira 


\section{Métodos}

O nível de sustentabilidade dos municípios da região turística da Costa do Cacau foi avaliado a partir do Índice de Desenvolvimento Sustentável para Municípios (IDSM), proposto por Martins e Cândido (2012) com escolha dos indicadores e especificidades pautados no desenvolvimento local. Os indicadores foram selecionados de acordo com as seguintes características: 1) disponibilidade, relevância e confiabilidade dos dados; 2) ser significativo para o objeto de estudo; 3) permitir o enfoque integrado e sistêmico; 4) utilizar variáveis mensuráveis; 5) ter uma metodologia bem definida, transparente e objetiva aos propósitos da investigação.

Para a definição das dimensões e variáveis utilizou-se como referência o IDS-Brasil realizado pelo IBGE em 2004, analisando a sustentabilidade a partir das dimensões social, ambiental, econômico e institucional (Quadro 1). Os dados para as análises foram obtidos nas seguintes bases: IBGE @ cidades (https://cidades.ibge.gov.br/), Atlas do Desenvolvimento Humano no Brasil (http:// atlasbrasil.org.br/2013/pt/perfil/), SEI - BA (Finanças Públicas) (https://www.sei.ba.gov.br/index. php?option=com_content\&view=article\&id=1167\&Itemid=388).

Quadro 1. Indicadores selecionados para as dimensões social, econômica, ambiental e institucional; fontes e ano dos indicadores que comporão o Índice de Desenvolvimento Sustentável (IDS).

\begin{tabular}{|c|c|c|c|}
\hline Dimensão & Indicador & Fonte & Ano \\
\hline \multirow{4}{*}{ Ambiental } & Arborização de vias públicas & IBGE & 2010 \\
\hline & $\%$ da população em domicílios com coleta de lixo & PNUD, IPEA, FJP & 2010 \\
\hline & Densidade demográfica & IBGE & 2010 \\
\hline & Esgotamento sanitário adequado & IBGE & 2010 \\
\hline \multirow{7}{*}{ Social } & \% da população em domicílios com energia elétrica & PNUD, IPEA, FJP & 2010 \\
\hline & $\begin{array}{l}\text { \% da população em domicílios com banheiro e água } \\
\text { encanada }\end{array}$ & PNUD, IPEA, FJP & 2010 \\
\hline & $\%$ de extremamente pobres & PNUD, IPEA, FJP & 2010 \\
\hline & $\%$ de pobres & PNUD, IPEA, FJP & 2010 \\
\hline & Taxa de escolarização de 6 a 14 anos de idade & IBGE & 2010 \\
\hline & Esperança de Vida ao nascer & PNUD, IPEA, FJP & 2010 \\
\hline & Mortalidade infantil & PNUD, IPEA, FJP & 2010 \\
\hline \multirow{5}{*}{ Econômica } & $\begin{array}{l}\text { Percentual da população com rendimento nominal mensal } \\
\text { per capita de até } 1 / 2 \text { salário mínimo }\end{array}$ & IBGE & 2010 \\
\hline & Renda per capita & PNUD, IPEA, FJP & 2010 \\
\hline & $\begin{array}{l}\% \text { de pessoas de } 18 \text { anos ou mais sem fundamental } \\
\text { completo e em ocupação informal }\end{array}$ & PNUD, IPEA, FJP & 2010 \\
\hline & PIB per capta & IBGE & 2010 \\
\hline & $\begin{array}{l}\text { Taxa de atividade (população economicamente ativa e } \\
\text { ocupada) - } 18 \text { anos ou mais }\end{array}$ & PNUD, IPEA, FJP & 2010 \\
\hline
\end{tabular}




\begin{tabular}{|l|l|l|c|}
\hline \multirow{5}{*}{ Institucional } & Despesas com educação & SEI/TCM-BA & 2010 \\
\cline { 2 - 4 } & Despesas com saúde & SEI/TCM-BA & 2010 \\
\cline { 2 - 4 } & Despesas com assistência social & SEI/TCM-BA & 2010 \\
\cline { 2 - 4 } & Despesas com urbanismo & SEI/TCM-BA & 2010 \\
\cline { 2 - 4 } & Despesas com gestão ambiental & SEI/TCM-BA & 2010 \\
\cline { 2 - 4 } & Despesas com transporte & SEI/TCM-BA & 2010 \\
\cline { 2 - 4 } & Despesas com saneamento básico & SEI/TCM-BA & 2010 \\
\cline { 2 - 4 } & Despesas com desporto e lazer & SEI/TCM-BA & 2010 \\
\cline { 2 - 4 } & Despesas com cultura & SEI/TCM-BA & 2010 \\
\hline
\end{tabular}

*PIB - Produto Interno Bruto

Uma vez definido os indicadores para cada uma das dimensões (Quadro1), procedeu-se a padronização (Equações 1 e 2) das variáveis selecionadas, de acordo com o método sugerido por Waquil et al. (2010), transformando os indicadores em índices, possibilitando a comparação entre as variáveis de unidades distintas e normalização dos dados em um número que varia de 0 a 1 , sendo que 0 representa a insustentabilidade e 1 sustentabilidade.

Levou-se em consideração a relação entre os indicadores com a sustentabilidade (positiva ou negativa) conforme a proposta metodológica de Sepúlveda (2005), que afirma haver necessidade de identificar o tipo de relação que cada indicador tem com o entorno geral. Em outras palavras, é necessário identificar se o indicador, ao aumentar seu valor, vai ocasionar em favorecimento ou desfavorecimento do desenvolvimento sustentável.

Conforme é identificada a relação positiva ou negativa são aplicadas as equações 1 e 2 , tornando possível a agregação de todos os índices. Essa relação é identificada da seguinte forma: positiva (quanto maior melhor e quanto menor pior) e negativa (quanto menor melhor e quanto maior pior), conforme o contexto da relação entre as variáveis e as dimensões (Martins e Cândido, 2012).

Para a padronização utilizou-se a equação (1), quando o indicador tem influência positiva e a equação (2), quando for negativa.

$$
\begin{aligned}
& x_{(+)}=\frac{o b s-m i ́ n}{m a ́ x-m i ́ n} \\
& x_{(-)}=\frac{m a ́ x-o b s}{m a ́ x-m i ́ n}
\end{aligned}
$$

Onde: $\mathrm{x}_{(.)}=$indicador padronizado, calculado para cada município; obs = valor do indicador em cada município; mín = valor mínimo do indicador de todos os municípios; máx = valor máximo do indicador de todos os municípios.

Após a padronização dos valores dos indicadores para os municípios, os índices gerados foram agregados nas dimensões definidas. Seguindo a metodologia proposta por Martins e Cândido (2012), o valor do índice de cada dimensão $\mathrm{j}(\mathrm{j}=1,2, \cdots, \mathrm{m})$, foi encontrado pela média aritmética 
simples dos índices de cada um dos temas i $(\mathrm{i}=1,2, \cdots, \mathrm{n})$, que compõe a dimensão analisada. Foram estabelecidos pesos iguais para as variáveis dentro de cada dimensão. A equação (3) é a média aritmética simples dos índices representativos de cada dimensão.

$$
D_{j}=\frac{\sum_{i=1}^{n} T_{i}}{n} ; \mathrm{j}=1,2,3, \ldots, \mathrm{m}
$$

Onde: $\mathrm{IDj}=$ índice da dimensão $\mathrm{j}(\mathrm{i}=1,2, \ldots, \mathrm{n}) ; \mathrm{Ti}$ = índice $\mathrm{i} ; \mathrm{m}=$ número de dimensões; $\mathrm{n}=$ número de índices.

A fórmula final do IDSM foi gerada através da média aritmética simples dos seus índices temáticos, equação (4).

$$
I D S M=\frac{I D S+I D E+I D I+I D A}{4}
$$

Onde: IDS = índice da dimensão social; IDE = índice da dimensão econômica; IDI = índice da dimensão institucional e; IDA = índice da dimensão ambiental.

$\mathrm{Na}$ Tabela 1 encontram-se os intervalos de valores do IDSM e os respectivos níveis de sustentabilidade, conforme a classificação de Martins e Cândido (2008). Com base nesses parâmetrosé possível classificar cada município quanto ao nível de sustentabilidade, bem como, a região estudada, estabelecendo assim comparação entre os mesmos.

Tabela 1. Classificação do nível de sustentabilidade dos municípios.

\begin{tabular}{cc}
\hline Índice $(\mathbf{0}-\mathbf{1})$ & Nível de Sustentabilidade \\
\hline $0,0000-0,2500$ & Crítico \\
$0,2501-0,5000$ & Alerta \\
$0,5001-0,7500$ & Aceitável \\
$0,7501-1,0000$ & Ideal \\
\hline
\end{tabular}

Fonte: Martins e Cândido (2008).

\section{Resultados e discussão}

A análise dos resultados obtidos (Tabela 2) revela que, dentre os municípios da região turística Costa do Cacau, somente Ilhéus se destaca por apresentar todos os índices classificados como ideal e aceitável, em relação ao nível de sustentabilidade. 
Tabela 2. Valores de Índice de Desenvolvimento Social (IDS), Índice de Desenvolvimento Econômico (IDE), Índice de Desenvolvimento Institucional (IDI), Índice de Desenvolvimento Ambiental (IDA) e Índice de Desenvolvimento Sustentável Municipal (IDSM) e nível de sustentabilidade dos municípios da região turística Costa do Cacau, Bahia.

\begin{tabular}{ccccccc}
\hline Municípios & IDS & IDE & IDI & IDA & IDSM & Nível de Sustentabilidade \\
\hline Canavieiras & 0,6990 & 0,3204 & 0,1908 & 0,7692 & 0,4949 & Alerta \\
Ilhéus & 0,9109 & 0,7613 & 0,8804 & 0,5845 & 0,7843 & Ideal \\
Itabuna & 0,9741 & 0,7149 & 0,5822 & 0,4913 & 0,6906 & Aceitável \\
Itacaré & 0,4937 & 0,4101 & 0,1589 & 0,4321 & 0,3737 & Alerta \\
Ipiaú & 0,8451 & 0,5879 & 0,1537 & 0,6769 & 0,5659 & Aceitável \\
Maraú & 0,3476 & 0,4247 & 0,0827 & 0,4190 & 0,3185 & Alerta \\
Pau Brasil & 0,3817 & 0,2623 & 0,0415 & 0,6843 & 0,3425 & Alerta \\
Santa Luzia & 0,3942 & 0,2526 & 0,0346 & 0,4228 & 0,2761 & Alerta \\
São José Da Vitoria & 0,4457 & 0,2290 & 0,0493 & 0,5470 & 0,3177 & Alerta \\
Una & 0,3394 & 0,4981 & 0,1279 & 0,5593 & 0,3812 & Alerta \\
Uruçuca & 0,6803 & 0,3605 & 0,0396 & 0,5549 & 0,4088 & Alerta \\
\hline
\end{tabular}

Dentre os municípios que apresentaram o IDSM no nível de alerta, Uruçuca e Canavieiras se destacaram pelos valores de IDA e IDS os enquadrarem nos níveis ideal e aceitável. Tal fato pode estar relacionado ao indicador de densidade demográfica em relação ao IDA, pois estes municípios apresentam 50,61 hab $/ \mathrm{km}^{2}$ e 24,37 hab/ $\mathrm{km}^{2}$, respectivamente, de acordo com o IBGE (2010). Estes dados de densidade demográfica são considerados baixos quando comparados com Itabuna, por exemplo, que apesar de apresentar o IDSM como aceitável, o IDA foi classificado como nível de alerta, pois este município apresenta densidade demográfica de $473,50 \mathrm{hab} / \mathrm{km}^{2}$ e área total $443,09 \mathrm{~km}^{2}$, enquanto Uruçuca e Canavieiras possuem $339,31 \mathrm{~km}^{2}$ e $1379,67 \mathrm{~km}^{2}$ de área total, respectivamente.

Dentre os municípios que apresentaram o IDSM no nível de alerta, apenas Uruçuca e Canavieiras se destacam pelos valores mais elevados de IDA e IDS, enquadrando-os nos níveis ideal e aceitável, respectivamente. Tal fato pode estar relacionado a densidade demográfica influenciando no IDA. Os dados de densidade demográfica de Uruçuca e Canavieiras são considerados baixos (50,6 e 24,4 $\mathrm{hab} / \mathrm{km}^{2}$, respectivamente, de acordo com o IBGE 2010), quando comparados com Itabuna, por exemplo. Apesar de apresentar o IDSM como aceitável, o IDA classificou o município de Itabuna como nível de alerta, pois o mesmo apresenta densidade demográfica de $473,5 \mathrm{hab} / \mathrm{km}^{2}$ em uma área total 443,09 $\mathrm{km}^{2}$. Uruçuca e Canavieiras apresentam densidade de 50,6 e 24,4 hab $/ \mathrm{km}^{2}$, em áreas de $339,31 \mathrm{~km}^{2}$ e $1.379,67 \mathrm{~km}^{2}$, respectivamente.

É importante destacar que todos os municípios analisados nesse estudo apresentaram cobertura de esgotamento sanitário abaixo de $82 \%$, sendo este um importante indicador elencado dentre os objetivos do desenvolvimento sustentável (ODS) da ONU (Organização das Nações Unidas). Cavinatto (2003) afirma que o acesso ao saneamento básico promove a saúde pública preventiva, reduzindo a busca pelos estabelecimentos de saúde ao diminuir a chance de contágio por diversas moléstias, sendo assim essencial na melhoria da qualidade de vida da população. 
A Política Nacional de Saneamento Básico (Lei no 11.445/2007) estabelece, em suas diretrizes, que todos os municípios brasileiros devem elaborar seus planos de saneamento básico, com prazo até 31 de dezembro de 2022, conforme decreto mais recente $n^{\circ}$ 10.203/2020. Apenas Itabuna, Ipiaú e Santa Luzia possuem o plano de saneamento básico, de acordo com dados do IBGE em 2017.

Ao comparar o IDS entre os municípios de Una e Uruçuca, classificados com desempenho turístico B e C (Ministério do Turismo 2020), respectivamente, nota-se uma substancial diferença no mesmo, apesar de Una apresentar melhor desenvolvimento econômico (IDS).

Comparando os dados de demografia (IBGE 2010) e destacando o indicador de população residente em área rural, o município de Una (37,66\%) apresenta uma porcentagem maior de população residente na área rural que Uruçuca $(20,46 \%)$, demonstrando ainda uma grande influência da agricultura (principalmente cultivo do cacau) no município de Una, enquanto Uruçuca apresentou uma taxa de urbanização maior em efeito do aumento das atividades turísticas o que, consequentemente, ocasionou a melhora dos indicadores sociais. Tal fato também pode ser relacionado à população imigrante que ocupou o município, sendo necessária a aplicação de um estudo mais aprofundado sobre a dinâmica de ocupação e imigração.

Ao analisar o desenvolvimento da atividade turística do município de Uruçuca a partir dos residentes locais, Santos e Ávila (2017) constataram que, apesar de integrado em áreas de proteção ambiental, o que requer do turismo um desenvolvimento com base em princípios sustentáveis, o nível de participação no planejamento municipal desse segmento é baixo. O Conselho Municipal de Turismo e Meio Ambiente (Comtuma) foi criado em 2003 pela Lei Municipal 367, no entanto, o nível de conhecimento a respeito dele no município é pequeno, sendo necessária a mobilização e ampliação do processo participativo para melhor desenvolvimento desta atividade, tendo como pressuposto que a participação de todos os interessados viabilizará condições de identificar os anseios dos diferentes segmentos, as potencialidades e fragilidades naturais, sociais e econômicas, bem como as dificuldades e as possibilidades que o turismo pode oferecer para o município.

Os municípios Itacaré e Maraú, que são classificados pelo desempenho turístico B (Ministério do Turismo 2020), apresentaram todos os índices em nível de sustentabilidade alerta ou crítico, evidenciando que é necessário melhorar a gestão da atividade turística nesses munícipios de acordo ao documento "Diretrizes para uma Política Nacional de Ecoturismo", lançado em 1994 pelo Ministério do Meio Ambiente, em parceria com a EMBRATUR e o IBAMA. O ecoturismo possui base no desenvolvimento sustentável, e por isso seus critérios são pautados na gestão socioambiental dos recursos naturais, enfatizando a importância do processo de planejamento multisetorial participativo, e levando em consideração as singularidades local e regional para a gestão pública, privada e comunitária do local em que se desenvolve essa atividade (Brasil 2010).

De acordo com Oliveira (2007), em 2005, Itacaré passou a receber na alta temporada 120 mil turistas, número seis vezes maior que sua população. O autor relata que o aumento do fluxo turístico foi responsável direto pelo surgimento de alguns problemas para a comunidade local, tais como, crescimento econômico desordenado, falta de infraestrutura, degradação ambiental, poluição, ocupação desordenada dos espaços (favelas), importação, vazamento da renda e especulação imobiliária.

Em relação a dimensão econômica dos munícipios Ilhéus, Itabuna, e Ipiaú, os dados representam os esforços dos gestores locais e da comunidade em criar uma base econômica que seja capaz de garantir a geração de riqueza, emprego, renda e consumo com equidade social. Apesar de 
apresentarem o nível de sustentabilidade classificado como ideal e aceitável, é oportuno e urgente a adoção de alternativas que promovam, de forma equitativa, a distribuição de renda da população.

Ao analisar o índice de Gini (PNUD; IPEA; FJP 2013), ferramenta utilizada para medir o grau de concentração de renda, Ilhéus $(0,58)$, Itabuna $(0,56)$, e Ipiaú $(0,69)$, apresentam valores que demonstram ainda uma grande desigualdade social, apesar desses municípios apresentarem um bom desempenho econômico. Corroborando com Pinheiro et al. (2019), ao analisarem a região metropolitana Vale do Paraíba e Litoral Norte constataram que os municípios apesar de demonstrarem bom desempenho econômico e Índice de Desenvolvimento Humano alto, isso não refletia na igualdade social, ou seja, a renda fica concentrada em apenas alguns grupos da sociedade, sendo necessário discutir sobre efetividade de planos de transferência de renda e outras ações voltadas para a diminuição da desigualdade social.

$\mathrm{Na}$ dimensão social os indicadores selecionados estão relacionados com o oferecimento de oportunidades e qualidade de vida da população, de forma que apresente uma visão dos aspectos mais importantes da realidade municipal, abordando informações relacionadas à saúde, educação, habitação e renda, podendo abordar também a subdimensão cultural. A análise do IDS dos municípios selecionados nessa pesquisa aponta para uma tendência que se estende para o estado da Bahia, no que diz respeito a falta de investimento no desenvolvimento da sociedade, má distribuição de recursos e, consequentemente, pobreza. Tratando-se de uma região vocacionada para o desenvolvimento do ecoturismo, que recebe investimentos nacionais e internacionais, é de extrema importância a gestão municipal com participação da sociedade local, para que o desenvolvimento econômico possa acontecer em conformidade com a sustentabilidade.

Com exceção do município de Ilhéus, Itabuna, e Ipiaú, todos os outros apresentaram o nível de sustentabilidade da dimensão ambiental como alerta, questão problemática para uma região que está inserida na zona litorânea do bioma da Mata Atlântica com a presença de várias Unidades de Conservação. Nesta direção, é necessário reavaliar de que forma está ocorrendo a gestão ambiental desses municípios e em que medida a legislação ambiental está sendo respeitada.

O índice de desenvolvimento institucional é uma importante ferramenta para a discussão do desenvolvimento sustentável municipal, pois tem como objetivo a caracterização das atividades governamentais na alocação dos recursos financeiros da arrecadação tributária em prol do benefício a do atendimento das demandas da sociedade. Entre os munícipios estudados, com exceção de Itabuna e Ilhéus, todos apresentaram nível crítico para o IDI, este resultado demonstra a necessidade de melhorar a gestão dos recursos de acordo com as particulares e necessidades de cada município, para que o desenvolvimento ocorra de forma igualitária, sustentável e com a participação da sociedade.

$\mathrm{Na}$ síntese dos indicadores levantados e formulação dos índices dos municípios, Ilhéus apresentou maior índice de desenvolvimento municipal sustentável. Acreditamos que isto se deva ao fato da cidade ser consideravelmente maior que as demais, e possivelmente, sua arrecadação e investimento também o sejam. Apesar de um resultado satisfatório na ótica do desenvolvimento sustentável, Ilhéus necessita melhorar bastante em alguns aspectos como, por exemplo, ampliação da rede de saneamento básico, melhoria da escolarização da população adulta e fluxo escolar na população jovem.

Em relação aos demais municípios que apresentaram o IDMS desfavorável, evidencia-se a necessidade urgente de implementação de políticas públicas em todas as dimensões tratadas a fim 
de elevar o grau de sustentabilidade e propiciar condições adequadas para a qualidade de vida da população atual e das futuras gerações.

\section{Considerações finais}

A aplicação do IDSM como ferramenta possibilitou a análise do nível de sustentabilidade dos municípios da região turística Costa do Cacau, e dessa forma, retratar as fragilidades e oportunidades, bem como os fatores que implicam maior influência na sustentabilidade dessas localidades. Esse tipo de informação é relevante para a formulação e implementação de políticas públicas para o desenvolvimento sustentável.

A metodologia aplicada nesse estudo também possibilitou a comparação entre as localidades, de forma que fosse caracterizada a falta de homogeneidade na região turística. Concluindo que, apesar do potencial das cidades estudadas para o desenvolvimento do ecoturismo, os municípios da região turística Costa do Cacau ainda estão se desenvolvendo de forma desigual, sendo necessária a aplicação de políticas adequadas às particularidades locais, porém tendo como referência a realidade de todo o território em análise.

Apesar de ser uma metodologia amplamente utilizada para tais analises, é importante destacar algumas limitações, primeiramente ao fato da escolha dos indicadores para cada dimensão, já que isso ocorre tendo como base outros trabalhos já realizados, podendo ocasionar a falta identidade em relação ao município estudado e a seleção de indicadores que não sejam relevantes para o estudo. Destaca-se também a problemática em relação a falta de atribuição de pesos para cada dimensão analisada, já que para cada região, a depender de suas características e especificidades, existe prioridades diferentes e graus de importância diferentes para cada dimensão. Dessa forma, é necessário para um estudo mais aprofundado que os indicadores sejam selecionados de forma participativa com os atores sociais e institucionais dos municípios.

Participação dos autores: ESO, FASR - coleta de dados, realização das análises, pesquisa bibliográfica, redação do manuscrito, AMSSM, FDRA, CCP - orientadores, revisão do manuscrito, EMS - pesquisa bibliográfica, revisão do manuscrito.

Aprovação ética e licenças: a obtenção da autorização pelo comitê de ética não se aplica a esta pesquisa.

Disponibilidade dos dados: a pesquisa não gerou base de dados para deposição, sendo utilizado dados coletados nas bases informadas na metodologia.

Fomento: não tem fontes de fomento para a pesquisa do artigo.

Conflito de Interesses: Não há conflito de interesse.

\section{Referências}

Aguiar PCB, Pires MM. 2019. A região cacaueira do sul do estado da Bahia, Brasil: crise e transformação. Cuadernos de Geografía: Revista Colombiana de Geografía 28 (1): 192-208. doi: 10.15446/rcdg.v28n1.67437.

Bahia. 2016. Desenvolvimento Territorial. Estudos de Potencialidades Econômicas: Território de Identidade Litoral Sul.

Brasil. 1988. Constituição. Constituição da República Federativa do Brasil. Brasília, DF: Senado Federal: Centro Gráfico. 
Brasil. 2010. Ministério do Turismo. Ecoturismo: orientações básicas. / Ministério do Turismo, Secretaria Nacional de Políticas de Turismo, Departamento de Estruturação, Articulação e Ordenamento Turístico, Coordenação Geral de Segmentação. 2. ed. - Brasília: Ministério do Turismo.

Brasil. 2007. Política Nacional De Saneamento Básico - Lei no 11.445, de 05 de janeiro de 2007, estabelece as diretrizes nacionais para o saneamento básico. Disponível em: < http://www.planalto.gov.br/ccivil_03/_ato2007-2010/2007/lei/ 111445.htm>. Acesso em: 22 set. 2020.

Bellen HMV. 2004. Indicadores de sustentabilidade - um levantamento dos principais sistemas de avaliação. Cadernos EBAPE.BR, Volume II - Número 1.

Carvalho KD, Coelho S, Bonfim NR. 2011. Turismo cultural e interpretativo na cidade de Ilhéus-Bahia: uma proposta de revitalização para a avenida Soares Lopes. Caderno Virtual de Turismo. Rio de Janeiro, v. 11, n.2., p.205-218.

Cavinatto VM. 2003. Saneamento básico: fonte de saúde e bem-estar. São Paulo: Ed. Moderna.

CMMAD - Comissão Mundial Sobre Meio Ambiente E Desenvolvimento. 1988. Nosso futuro comum. Rio de Janeiro: Fundação Getulio Vargas.

Frainer DM, Souza CC, Neto JFR, Castelão RA. 2017. Uma aplicação do Índice de Desenvolvimento Sustentável aos municípios do estado de Mato Grosso do Sul. Interações (campo Grande), Campo Grande, v. 18, n. 2, p.145-156. Universidade Católica Dom Bosco. http://dx.doi.org/10.20435/inter.v18i2.1524. Disponível em: <http://www.interacoes. ucdb.br/article/view/1524/pdf>. Acesso em: 13 jan. 2020.

IBGE - Instituto Brasileiro de Geografia e Estatística. 2010. Censo Demográfico, 2010. Disponível em: <www.cidades. ibge.gov.br>. Acesso em: 14 jan. 2020.

IBGE - Instituto Brasileiro de Geografia e Estatística. 2004. Indicadores de Desenvolvimento Sustentável: Brasil, 2004. Diretoria de Geociências (Estudos e Pesquisas). Rio de Janeiro: IBGE.

IBGE - Instituto Brasileiro de Geografia e Estatística. Cidades. Disponível em: < https://cidades.ibge.gov.br/brasil/ba/ itabuna/pesquisa/38/47001? localidade1=291360\&tipo=ranking\&indicador=46997>. Acesso em: 22 set. 2020.

Leff E. 2004. Saber Ambiental: sustentabilidade, racionalidade, complexidade, poder. 3 ed. Petrópolis: Vozes.

Malheiros TF, Phlippi Jr. A, Coutinho SMV. 2008. Agenda 21 nacional e indicadores de desenvolvimento sustentável: contexto brasileiro. Saude soc., São Paulo, v. 17, n. 1, p. 7-20. Disponível em: <http://www.scielo.br/scielo.php?script=sci_ arttext\&pid=S010412902008000100002\&lng=en\&nrm=iso >. Acesso em: 22 set. 2020.

Martins MF, Cândido GA. 2012. Índices de desenvolvimento sustentável para localidades: uma proposta metodológica de construção e análise. Revista de Gestão Social e Ambiental - RGSA, São Paulo, v. 6, n. 1, p. 03- 19.

Martins MF, Cândido GA. 2008. Metodologia para Construção e Análise de Índices de Desenvolvimento Sustentável: uma aplicação no Estado da Paraíba. João Pessoa - PB: Edições SEBRAE.

Ministério do Turismo. MAPA DO TURISMO 2019 - 2021. Disponível em: < http://www.mapa.turismo.gov.br/mapa/ init.html\#/home>. Acesso em: 21 set. 2020.

Oliveira ES. 2007. Impactos socioambientais e econômicos do turismo e as suas repercussões no desenvolvimento local: o caso do Município de Itacaré - Bahia. Revista Internacional de Desenvolvimento Local. Vol. 8, N. 2, p. $193-202$.

Pinheiro W, Vieira ET, Oliveira EAAQ. 2019. As relações entre o índice de gini com o índice de desenvolvimento humano e o produto interno bruto dos municípos da RMVPLN sob a ótica do desenvolvimento regional. Revista Humanidades e Inovação, v.6, n.12.

PNUD - Programa Das Nações Unidas Para O Desenvolvimento; IPEA - Instituto De Pesquisa Econômica E Aplicada; FJP - Fundação João Pinheiro. 2013. Atlas de desenvolvimento humano do Brasil de 2013. Disponível em: < http:// atlasbrasil.org.br/2013/pt/> . Acesso em: 22 set. 2020. 
Rezende GBM, Fagundes EAA. 2017. Índice de Desenvolvimento Sustentável de Primavera do Leste - MT, baseado no modelo de Martins e Cândido (2008). Revista Estudos e Pesquisas em Administração, [S.1.], v. 1, n. 1, p. 91-107, ISSN 2594-7559. Disponível em: <http://periodicoscientificos.ufmt.br/ojs/index.php/repad/article/view/5806/3870>. Acesso em: 14 jan. 2020.

Santos MS, Avila MA. 2017. Planejamento participativo: uma análise do turismo de Uruçuca (BA) a partir da ótica dos residentes. Caderno Virtual de Turismo. Rio de Janeiro, v. 17, n. 1, p. 30-45.

Sepúlveda S. 2005. Desenvolvimento microrregional sustentável: métodos para planejamento local. Brasília: IICA, p. 239.

Soares SP, Soares AP, Nunes FR. 2014. O Nordeste Brasileiro Na Contextualidade Econômica Da Costa Do Cacau. Revista Perspectiva Geográfica. v.9, n.11.

SEI - Superintendência De Estudos Econômicos E Sociais. Finanças Públicas. Disponível em: < https://www.sei.ba.gov. br/index.php?option=com_content\&view=article\&id=1167\&Itemid=388>. Acesso em: 20 set. 2020.

Waquil P, Schneider S, Filippi E, Rückert A, Rambo A, Radomsky G, Conterato M, Specht S. 2010. Avaliação de desenvolvimento territorial em quatro territórios rurais no Brasil. Redes, v. 15, n. 1, p. 104-127. 